\title{
On shape of hyperspaces
}

Dedicated to professor Kiiti Morita for his 60th birthday

by

Y. Kodama (Ibaragi), S. Spiéz (Warszawa) and T. Watanabe (Tokyo)

Abstract. For a compact space $X$ denote by $2^{X}$ the hyperspace consisting of all non empty closed subsets of $X$ and by $C(X)$ the hyperspace consisting of all non empty connected closed subsets of $X$ with finite topology. Then it is proved that $\operatorname{Sh}\left(2^{X}\right)=\operatorname{Sh}\left(2^{\square X}\right)$ and $\operatorname{Sh}(C(X))=\operatorname{Sh}(\square X)$, where $\square X$ is the decomposition space of $X$ consisting of all components. As a consequence, if $X$ is connected then $\operatorname{Sh}\left(2^{X}\right)$ and $\operatorname{Sh}(C(X))$ are trivial. Also for any compact spaces $X$ and $Y$ such that both $\square X$ and $\square Y$ are countably infinite, we have $\operatorname{Sh}\left(2^{X}\right)=\operatorname{Sh}\left(2^{X}\right)$. If $X(n)$ denotes the $n$th symmetric product of $X$, then it is proved that $\operatorname{Sh}(X) \geqslant \operatorname{Sh}(Y)$ means $\operatorname{Sh}(X(n)) \geqslant \operatorname{Sh}(Y(n))$. Hence if $X$ is an ASR(ANSP) so is $X(n)$

\$ 1. Introduction. Let $X$ be a compact Hausdorff space. We denote by $2^{X}$ the hyperspace with finite topology consisting of all non empty closed subsets of $X$ and by $C(X)$ the hyperspace with finite topology consisting of all non empty closed con列 nected subsets of $X$. Let $\square X$ be the decomposition space of $X$ consisting or all com$=\operatorname{Sh}(\square X)$. Here by $\operatorname{Sh}(X)$ is meant the shape of $X$ (cf. Borsuk [2], Mardešić and Segal $[8,9]$ and Mardešić [11]). As a consequence the following corollaries are obtained.

(1) If $X$ is connected, then $\operatorname{Sh}\left(2^{X}\right)$ and $\operatorname{Sh}(C(X))$ are trivial.

(2) If $\square X$ and $\square Y$ are metrizable and infinite, then $\operatorname{Sh}\left(2^{X}\right) \equiv \operatorname{Sh}\left(2^{Y}\right)$. (Here we mean by $\operatorname{Sh}(A) \equiv \operatorname{Sh}(B)$ that both the relations $\operatorname{Sh}(A) \leqslant \operatorname{Sh}(B)$ and $\operatorname{Sh}(A) \geqslant \operatorname{Sh}(B)$ hold.)

(3) If both. $\square X$ and $\square Y$ are countably infinite, then $\operatorname{Sh}\left(2^{X}\right)=\operatorname{Sh}\left(2^{X}\right)$.

For a positive integer $n$, let $X(n)$ be the $n$th symmetric product of $X$. We shall show that if $\operatorname{Sh}(X)=\operatorname{Sh}(Y)$ then $\operatorname{Sh}(X(n))=\operatorname{Sh}(Y(n))$.

Throughout this paper all of spaces are Hausdorff and maps are continuous. By an AR-space and an ANR-space we mean always those for metric spaces.

82. Hyperspaces of the inverse limit space. Let $X$ be a space. We denote by $2^{X}$ the set of all nonempty closed subsets of $X$, by $C(X)$ the set of all non empty closed connected subsets of $X$ and by $X(n), n$ a positive integer, the set of all non empty subsets consisting of at most $n$ points. We consider $C(X)$ and $X(n)$ as subsets of $2^{X}$. 
Let $\left\{U_{j}: j=1, \ldots, k\right\}$ be a finite collection of open sets of $X$. Denote by $\left\langle U_{1}, \ldots, U_{k}\right\rangle$ the set $\left\{F \in 2^{X}: F \subset \bigcup_{i=1}^{k} U_{j}\right.$ and $F \cap U_{j} \neq \varnothing$ for each $\left.j\right\}$. The finite topology of $2^{X}$ is the one generated by collections of the form $\left\langle U_{1}, \ldots, U_{k}\right\rangle$ with $U_{1}, \ldots, U_{k}$ open sets of $X$. (See Michael [13, Def. 1.7].) Throughout this paper we assume that $2^{X}$ has the finite topology and $C(X)$ and $Y(n)$ are subspaces of $2^{X}$.

Let $X$ and $Y$ be compact spaces and let $f: X \rightarrow Y$ be a continuous map. Define $f_{*}: 2^{X} \rightarrow 2^{Y}$ by $f_{*}(F)=f(F)$ for $F \in 2^{X}$. Then by $[13,5.10] f_{*}$ is continuous and $f_{*}\left(C^{\prime}(X)\right) \subset C(Y)$ and $f_{*}(X(n)) \subset Y(n)$. We say that $f_{*}$ is a map induced by $f$.

LEMMA 1. Let $f, g: X \rightarrow Y$ be maps of a compact space $X$ into a space $Y$. If $f \simeq g$ then $f_{*} \simeq g_{*}, f_{*}\left|C(X) \simeq g_{*}\right| C(X)$ in $C(Y)$ and $f_{*}\left|X(n) \simeq g_{*}\right| X(n)$ in $Y(n)$.

Proof. Let $H: X \times I \rightarrow Y$ be a homotopy connecting $f$ and $g$. Define $H^{\prime}: 2^{X} \times I \rightarrow 2^{Y}$ by $H^{\prime}(F, t)=H(F \times\{t\})$ for $F \in 2^{X}$ and $t \in I$. It is easy to show that $H^{\prime}$ is continuous, $H^{\prime}(C(X) \times I) \subset C(Y)$ and $H^{\prime}(X(n) \times I) \subset Y(n)$. Since $H^{\prime}(F, 0)=f_{*}(F)$ and $H^{\prime}(F, 1)=g_{*}(F)$ for $F \in 2^{X}$, the lemma is proved.

Let $\left\{X_{\alpha}, \pi_{\alpha}^{\beta}, \Omega\right\}$ be an inverse system consisting of compact spaces $X_{\alpha}$ and projections $\pi_{\alpha}^{\beta}: X_{\beta} \rightarrow X_{\alpha}, \alpha<\beta, \alpha, \beta \in \Omega$, where $\Omega$ is a directed set. Then $\left\{2^{X_{\alpha}}, \pi_{\alpha *}^{\beta}\right\},\left\{C\left(X_{\alpha}\right), \pi_{\alpha *}^{\beta} \mid C\left(X_{\beta}\right)\right\}$ and $\left\{X_{\alpha}(n), \pi_{\alpha *}^{\beta} \mid X_{\beta}(n)\right\}$ form inverse systems over $\Omega$, where $\pi_{\alpha *}^{\beta}: 2^{X_{\beta}} \rightarrow 2^{X_{\alpha}}$ is induced by $\pi_{\alpha}^{\beta}$. The following lemma was essentially proved by Segal [19].

LEMMA 2. Let $X=\varliminf_{\alpha} X_{\alpha}$. Then $2^{X}=\varliminf 2^{X_{\alpha}}, \quad C(X)=\varliminf C\left(X_{\alpha}\right)$ and $X(n)=\varliminf_{\alpha}(n)$.

Proof. Let $\pi_{\alpha}: X \rightarrow X_{\alpha}, \alpha \in \Omega$, be the projection. Consider the maps $\pi_{\alpha *}: 2^{X} \rightarrow 2^{X_{\alpha}}, \alpha \in \Omega$. Since $\pi_{\alpha}^{\beta} \pi_{\beta}=\pi_{\alpha}$ for $\alpha<\beta, \pi_{\alpha *}^{\beta} \pi_{\beta *}=\pi_{\alpha *}$ and hence the collection of maps $\left\{\pi_{\alpha *}, \alpha \in \Omega\right\}$ defines uniquely a continuous map $\pi_{*}: 2^{X} \rightarrow \varliminf$ $2^{X_{\alpha}}$. Obviously $\pi_{*}(C(X)) \subset \varliminf C\left(X_{\alpha}\right)$ and $\pi_{*}(X(n)) \subset \varliminf X_{\alpha}(n)$. Let $x=\left\{F_{\alpha}: F_{\alpha} \subset X_{\alpha}, \alpha \in \Omega\right\}$ be a point of $\varliminf^{X_{\alpha}}$. Then $\pi_{\alpha *}^{\beta}\left(F_{\beta}\right)=F_{\alpha}$ so that $\pi_{\alpha}^{\beta}\left(F_{\beta}\right)=F_{\alpha}$ for each $\beta>\alpha$. Since $\left\{F_{\alpha}, \pi_{\alpha}^{\beta} \mid F_{\beta}\right\}$ forms an inverse system of compact sets with onto bonding maps, $F_{x}=\varliminf F_{\alpha} \in 2^{X}$ and $\pi_{\alpha}\left(F_{x}\right)=F_{\alpha}$ for each $\alpha \in \Omega$. If $x \in \varliminf C\left(X_{\alpha}\right)\left(\right.$ resp. $\left.x \in \varliminf X_{\alpha}(n)\right)$ then $F_{x} \in C(X)$ (resp. $F_{x} \in X(n)$ ). Obviously $\pi_{*}\left(F_{x}\right)=x$. Thus $\pi_{*}$ is onto. Similary it is proved $\pi_{*}$ is one-to-one. The lemma is obtained by the compactness of $2^{\dot{X}}, C(X)$ and $X(n)$.

LEMMA 3. Let $f: X \rightarrow Y$ be a map from a compact space $X$ into a space $Y$. Let $A=\left\{y_{j} ; j=1, \ldots, k\right\}$ be a finite set of $Y$. Then $f_{*}^{-1}(A)=\prod_{j=1}^{k} 2^{f^{-1}\left(y_{j}\right)}$.

The lemma follows from the definition of the map $f_{*}$ induced by $f$.

The following lemmas were obtained by Wojdysławski [20, Théorème II and $\left.\mathrm{II}_{m}\right]$ and Ganea [3, Korollar].

LEMMA 4 (Wojdysławski). If $X$ is a compact connected ANR-space then $2^{X}$ and $C(X)$ are compact AR-spaces.
LEMMA 5 (Ganea). If $X$ is a finite dimensional compact ANR, then $X(n)$ is a compact ANR-space.

$\S$ 3. Shape of hyperspaces. For a space $X$, we mean by $\operatorname{Sh}(X)$ the shape of $X$ defined by Mardešić [11]. By Mardešić [11, Th. 6.8 and $\S 7$ ] this shape is equal to one defined by Borsuk [2] if spaces are compact metric and one defined by Mardešić and Segal [8] if spaces are compact. For a space $X, \square X$ denotes the decomposition space defined by the decomposition consisting of all components of $X$. If $f: X \rightarrow Y$ is a map, then a map $\square f: \square X \rightarrow \square Y$ satisfying $\pi_{Y} \cdot f=\square f \cdot \pi_{X}$ is uniquely defined, where $\pi_{X}$ is the decomposition (quotient) map from $X$ onto $\square X$.

THEOREM 1. Let $X$ be compact and let $\pi_{X}: X \rightarrow \square X$ be the decomposition map. Then each of maps $\pi_{X *}: 2^{X} \rightarrow 2^{\square X}$ and $\pi_{X *}: C(X) \rightarrow C(\square X)=\square X$ induces a shape equivalence. In particular, $\operatorname{Sh}\left(2^{X}\right)=\operatorname{Sh}\left(2^{\square X}\right)$ and $\operatorname{Sh}(C(X))=\operatorname{Sh}(\square X)$.

We need the following lemma.

LEMMA 6. If $X$ is a compact metric ANR, then the map $\left(\pi_{X}\right)_{*}: 2^{X} \rightarrow 2^{\square X}$ is a homotopy equivalence.

Proof. Let $y=\left\{y^{1}, y^{2}, \ldots, y^{k}\right\} \in 2^{\square x}$. Then by Lemmas 3 and $4,\left(\pi_{X}\right)_{*}^{-1}(y)$ $=\prod_{i=1}^{k} 2^{\pi_{\bar{x}}^{-1}\left(y^{\prime}\right)}$ is a compact metric AR-space. It is easy to see that for any different $y, y^{\prime} \in 2^{\square x},\left(\pi_{x}\right)_{*}^{-1}(y)$ and $\left(\pi_{X}\right)_{*}^{-1}\left(y^{\prime}\right)$ are disjoint and $2^{\square x}$ is finite. Thus $\left(\pi_{X}\right)_{*}$ is a homotopy equivalence.

Proof of Theorem 1. We shall prove the first part of Theorem 1 (the proof of the second part is similar, only simpler). Let $\underline{X}=\left\{X_{\alpha}, \pi_{\alpha}^{\beta}, \Omega\right\}$ be an ANR-system associated with a compact space $X$. Then it is easy to prove that

$$
\square X=\varliminf \underline{\lim }\left\{\square X_{\alpha}, \square \pi_{\alpha}^{\beta}, \Omega\right\} .
$$

By Lemma 2 we have $2^{X}=\varliminf\left\{2^{X_{\alpha}},\left(\pi_{\alpha}^{\beta}\right)_{*}, \Omega\right\}$ and $2^{\square X}=\varliminf\left\{2^{\square X},\left(\square \pi_{\alpha}^{\beta}\right)_{*}, \Omega\right\}$. For any $\alpha, \beta \in \Omega, \alpha \leqslant \beta$, the following diagram commutates

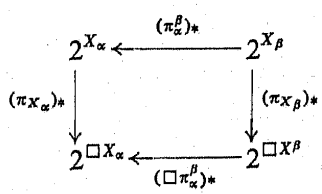

because $\pi_{X_{\alpha}} \pi_{\alpha}^{\beta}=\left(\square \pi_{\alpha}^{\beta}\right) \pi_{X_{\beta}}$. Thus the system of maps $\left\{\left(\pi_{X_{\alpha}}\right)\right\}_{\alpha \in \Omega}$ is the map from the system. $\left\{2^{X^{\alpha}},\left(\pi_{\alpha}^{\beta}\right)_{*}, \Omega\right\}$ to the system $\left\{2^{\square X_{\alpha}},\left(\square \pi_{\alpha}^{\beta}\right)_{*}, \Omega\right\}$, which is a homotopy equivalence in the sense of Mardesic (because every $\left(\pi_{X_{\alpha}}\right)_{*}$ is a homotopy equivalence). It is easy to see that the map $\left(\pi_{X}\right)_{*}: 2^{X} \rightarrow 2^{\square X}$ is the inverse limit of the map (of systems) $\left\{\left(\pi_{X_{\alpha}}\right)_{*}\right\}_{\alpha \in \Omega}$. Thus $\left(\pi_{X}\right)_{*}$ is the shape equivalence.

Next we shall give alternative proof of Theorem 1 . We start with the following lemma. 
LEMMA 7. Let $X$ be a paracompact space. Suppose that there is a closed map from $X$ onto a space $Y$ with $\operatorname{dim} Y=0$ such that for each $y \in Y f^{-1}(y)$ is of trivial shape. Let $H$ be an ANR-space.

(3.1) If $g: X \rightarrow H$, then there is a map $g^{\prime}: Y \rightarrow H$ such that $g^{\prime} f \simeq g$ and the homotopy class of $g^{\prime}$ is determined uniquely by the homotopy class of $g$.

(3.2) Let $g, h: X \rightarrow H$. Then $g \simeq h$ if and only if $\pi_{H} g=\pi_{H} h: X \rightarrow \square H$, where $\pi_{H}: H \rightarrow \square H$ is the decomposition map.

Proof. Let $g: X \rightarrow H$. Take any point $y \in Y$. Since $f^{-1}(y)$ is connected, $g\left(f^{-1}(y)\right)$ is connected. Let $H_{y}$ be the component of $H$ containing $g\left(f^{-1}(y)\right)$. Then $H_{y}$ is an ANR-space. Since $f^{-1}(y)$ is of trivial shape and $X$ is paracompact, it is easy to show that there is an open neighborhood $U_{y}$ of $f^{-1}(y)$ in $X$ and a homotopy $h_{y}: U_{y} \times I \rightarrow H_{y}$ such that

(3.3) $h_{y}(x, 0)=g(x)$ and $h_{y}(x, 1)=p_{y}\left(=a\right.$ point of $\left.H_{y}\right)$ for each $x \in U_{y}$.

This is done by using a bridge map theorem (see for example [4, Theorem 5]). Put $V_{y}=Y-f\left(X-U_{y}\right), y \in Y$. By the closedness of $f Y$ is paracompact and $\left\{V_{y}: y \in Y\right\}$ forms an open cover of $Y$. Since $\operatorname{dim} Y=0$, there is a locally finite open cover $\mathscr{W}=\left\{W_{\alpha}: \alpha \in \Omega\right\}$ such that order of $\mathscr{W}=1$ and $\mathscr{W}$ refines $\left\{V_{y}: y \in Y\right\}$. For each $\alpha \in \Omega$, choose a point $y_{\alpha}$ of $Y$ such that $W_{\alpha} \subset V_{y_{\alpha}}$. Define $g^{\prime}: Y \rightarrow H$ by $g^{\prime}(y)=p_{y_{\alpha}}$ for $y \in W_{\alpha}, \alpha \in \Omega$ (cf. (3.3)). Since order of $\mathscr{W}=1, g^{\prime}$ is continuous. Since $\left\{f^{-1}\left(W_{\alpha}\right) ; \alpha \in \Omega\right\}$ forms a locally finite open cover of $X$ whose order $=1$, and $g^{\prime} f\left|f^{-1}\left(W_{\alpha}\right) \simeq g\right| f^{-1}\left(W_{\alpha}\right)$ for each $\alpha \in \Omega$ by (3.3) and the definition of $g^{\prime}$, we know $g^{\prime} f \simeq g$. This completes the proof of the first part of (3.1). Next, let us prove (3.2). Since $\square H$ is a discrete space by the local connectedness of $H$, it follows that $g \simeq h$ implies $\pi_{H} g=\pi_{H} h$. Suppose that $\pi_{H} g=\pi_{H} h$. Let $g^{\prime}$ and $h^{\prime}$ be maps of $Y$ into $H$ constructed for $g$ and $h$ in the proof of (3.1) respectively. Let $\mathscr{W}_{g}$ and $\mathscr{W}_{h}$ be locally finite open covers of $Y$ used for the constructions of $g^{\prime}$ and $h^{\prime}$. Take a locally finite open refinement $\mathscr{W}$ of $\mathscr{W}_{g} \wedge \mathscr{W}_{h}$ such that order of $\mathscr{W}=1$. From $\pi_{H} g=\pi_{H} h$ and the definition of $g^{\prime}$ and $h^{\prime}$, we know for each $W \in \mathscr{W}$ two points $g^{\prime}(W)$ and $h^{\prime}(W)$ belong to the same component of $H$. Since each component of $H$ is arcwise connected, $g^{\prime} \simeq h^{\prime}$ and hence $g \simeq f g^{\prime} \simeq f h^{\prime} \simeq h$. This completes the proof of (3.2). The second half of (3.1) is a consequence of (3.2).

By Lemma 7 we obtain the following theorem. In case $X$ and $Y$ are metrizable and $X$ is finite dimensional, it is a consequence of [6, Theorem. 1]. Note that we do not assume the finite dimensionality of $X$ in the theorem.

THEOREM 2. Assume that $X, Y$ and f satisfy the same hypothesis as in Lemma 7. Then the shaping $f: X \rightarrow Y$ induced by $f$ (cf. [11]) is a shape equivalence. In particular $\operatorname{Sh}(X)=\operatorname{Sh}(Y)$.

Proof. We have to construct a shaping $\varphi: Y \rightarrow X$ such that $\varphi \tilde{f}=\tilde{1}_{X}$ and $\tilde{f}_{\varphi}=\tilde{1}_{Y}$, where $\tilde{1}_{X}$ and $\tilde{1}_{Y}$ are the shapings induced by the identities $1_{X}: X \rightarrow X$ and $1_{Y}: Y \rightarrow Y$. For a map $g$ of $X$ into an ANR-space $K$, define $\varphi(g)=g^{\prime}$, where $g^{\prime}$ is a map of $Y$ into $K$ constructed for $g$ in Lemma 7 (3.1). To show $\varphi$ is a shaping, let $L$ be an
ANR-space and let $\xi: K \rightarrow L$ and $h: X \rightarrow L$ be maps such that $\xi g \simeq h$. Since $\pi_{L} \xi \varphi(g) f=\pi_{L} \xi g=\pi_{L} h=\pi_{L} \varphi(h) f$, we have $\xi \varphi(g) f \simeq \varphi(h) f$ by (3.2). Hence, by the uniqueness of $g^{\prime}$ in (3.1), we know $\xi \varphi(g) \simeq \varphi(h)$. This implies that $\varphi$ is a shaping. It is easy to prove by (3.1) and the definition of $\tilde{f}$ that $\varphi \tilde{f}=\tilde{1}_{X}$ and $\tilde{f} \varphi=\tilde{1}_{Y}$. This completes the proof.

EXAMPLE 1. Consider the following sets in the plane $R^{2}: A_{0}=\{(0,0)\}$,

$$
A_{i}=\left\{(x, y): x \geqslant 0,(x-1)^{2}+y^{2}=(1+1 / i)^{2}\right\},
$$

$i=1,2, \ldots, X=\bigcup_{i=0}^{\infty} A_{i} ; Y=\{(0,0)\} \cup\{(0,1 / i) ; i=1,2, \ldots\}$. We define $f: X \rightarrow Y$ by $f\left(A_{0}\right)=(0,0)$ and $f\left(A_{i}\right)=(0,1 / i), i=1,2, \ldots$ Next, let

$$
A_{0}^{\prime}=\left\{(x, y): x \neq 2,(x-1)^{2}+y^{2}=1\right\}
$$

and put $X^{\prime}=A_{0}^{\prime} \cup \bigcup_{i=1}^{\infty} A_{i}$. Define $g: X^{\prime} \rightarrow Y$ by $g\left(A_{i}\right)=(0,1 / i), i>0$ and $g\left(A_{0}^{\prime}\right)=(0,0)$. Then $f$ and $g$ are continuous and open maps and for each $y \in Y f^{-1}(y)$ and $g^{-1}(y)$ are a point or an open interval or a closed interval. However, since $\check{H}^{1}(X)$ and $\check{H}^{1}\left(X^{\prime}\right)$ are both infinite groups and $\check{H}^{1}(Y)=0$, each of $\tilde{f}$ and $\tilde{g}$ is not a shape equivalence, where $\check{H}^{*}$ is the integral Čech cohomology. We know that $X^{\prime}$ is locally compact. These examples are shown that we can not replace the closedness of a map $f$ in Theorem 2 by the condition (i) or (ii):

(i) $f$ is open and for each $y \in Y f^{-1}(y)$ is compact;

(ii) $X$ is locally compact and $f$ is open.

(Note that if two conditions (i) and (ii) are satisfied then $f$ becomes a closed map.)

Alternative proof of Theorem 1. Since $X$ is compact, $\square X$ is a compact space and $\operatorname{dim} \square X=0$ by Ponomarev [16]. Hence $2^{\square X}$ is compact and $\operatorname{dim} 2^{\square X}=0$. Therefore by Theorem 2 , it is enough to prove that for each point $y$ of $2^{\square x}$ or $C(\square X)=\square X,\left(\pi_{X}\right)_{*}^{-1}(y)$ is of trivial shape. However it is easily proved by Lemmas 2 and 6.

There are several corollaries of Theorem 1 . The first concerns an absolute shape retract (ASR) and an absolute neighborhood shape retract (ANSR) (see [10] for the definitions.)

Corollary 1. Let $X$ be compact. Then:

(3.4) $2^{X}$ and $C(X)$ are ASR (equivalently of trivial shape [10, Theorem 4]) if and only if $X$ is connected.

(3.5) $2^{X}$ and $C(X)$ are ANSR if and only if $X$ has a finite number of components.

Proof. The if part is an immediate consequence of Theorem 1 because $\square X$ is a singleton or a finite set. Next, let us prove the only if part of (3.5). Then by Mardešic $\left[10\right.$, Corollary 2] there exists an ANR (compact) $Y$ such that $\operatorname{Sh}\left(2^{X}\right) \leqslant \operatorname{Sh}(Y)$ or 
$\operatorname{Sh}(C(X)) \leqslant \operatorname{Sh}(Y)$. Since for compact spaces $A$ and $B \quad \operatorname{Sh}(A) \leqslant \operatorname{Sh}(B)$ implies $\operatorname{Sh}(\square A) \leqslant \operatorname{Sh}(\square B)$, we have $\operatorname{Sh}\left(\square 2^{X}\right) \leqslant \operatorname{Sh}(\square Y)$ or $\operatorname{Sh}(\square C(X)) \leqslant \operatorname{Sh}(\square Y)$. Also it is easy to know that $\operatorname{Sh}\left(\square 2^{X}\right)=\operatorname{Sh}\left(2^{\square X}\right)$ and $\operatorname{Sh}(\square C(X))=\operatorname{Sh}(C(\square X))$. By Theorem 1 we have $\operatorname{Sh}\left(2^{\square X}\right) \leqslant \operatorname{Sh}(\square Y)$ or $\operatorname{Sh}(\square X) \leqslant \operatorname{Sh}(\square Y)$. Since $Y$ is an ANR (compact), $\square Y$ is a finite set. Since $\operatorname{dim} 2^{\square X}=\operatorname{dim} \square X=0$, by Mardešić and Segal [8, Theorem 20] we can conclude $\square X$ is a finite set. The proof of (3.4) is similar.

Corollary 2. For every compact space $X, 2^{X}$ and $C(X)$ are movable.

Proof. By Theorem 1 we know that each of $2^{X}$ and $C(X)$ has the same shape type as a 0 -dimentional compact space. The corollary follows from Mardešic and Segal [7, Example 2].

Corollary 3. Let $X$ and $Y$ be compact spaces. If $\operatorname{Sh}(X) \geqslant \operatorname{Sh}(Y)$ (resp. $\operatorname{Sh}(X)=\operatorname{Sh}(Y))$, then $\operatorname{Sh}\left(2^{X}\right) \geqslant \operatorname{Sh}\left(2^{X}\right)$ and $\operatorname{Sh}(C(X)) \geqslant \operatorname{Sh}(C(Y))$ (resp. $\operatorname{Sh}\left(2^{X}\right)$ $=\operatorname{Sh}\left(2^{Y}\right)$ and $\operatorname{Sh}(C(X))=\operatorname{Sh}(C(Y))$ ).

Proof. By the proof of Lemma 6 and Mardešic and Segal [8, Theorem 20], we know that any shaping $\varphi: X \rightarrow Y$ determines uniquely a continuous map $f_{\varphi}: \square X \rightarrow \square Y$ such that $\tilde{f}_{\varphi} \tilde{\pi}_{X}=\tilde{\pi}_{Y} \varphi$, where $\tilde{g}$ denotes the shaping determined by a map $g$. If $\psi: Y \rightarrow X$ is a shaping such that $\psi \varphi=\tilde{1}_{X}$, where $1_{X}$ is the identity of $X$, then the map $f_{\psi \varphi}=f_{\psi} f_{\varphi}: \square X \rightarrow \square X$ is the identity. Thus $f_{\psi \varphi *}=f_{\psi *} f_{\varphi *}: 2^{\square X} \rightarrow 2^{\square X}$ is the identity so that $\operatorname{Sh}\left(2^{\square X}\right) \leqslant \operatorname{Sh}\left(2^{\square Y}\right)$. The corollary follows from Theorem 1 .

Corollary 4. If $|\square X|=|\square Y|=s_{0}$, then $\operatorname{Sh}\left(2^{X}\right)=\operatorname{Sh}\left(2^{Y}\right)$, where $|Z|$ denotes the cardinality of $Z$.

Proof. Since $\square X$ and $\square Y$ are compact, it follows from Arhangel'skiř [1] that $\square X$ and $\square Y$ are metrizable. Since both $\square X$ and $\square Y$ have dense sets of isolated points, $2^{\square X}$ and $2^{\square Y}$ are homeomorhic by Pełczyński [15]. Thus $\operatorname{Sh}\left(2^{X}\right)=\operatorname{Sh}\left(2^{\square X}\right)$ $=\operatorname{Sh}\left(2^{\square Y}\right)=\operatorname{Sh}\left(2^{Y}\right)$.

Denote by $\mathscr{M}$ the class of all compact spaces $X$ such that $\square X$ is metrizable. We note that the hypothesis of Corollary 4 can be replaced by the following: $X, Y \in \mathscr{M}$ and both $\square X$ and $\square Y$ have countable infinite dense sets of isolated points (cf. Pełczyński [15]).

Corollary 5. If $X, Y \in \mathscr{M}$, then $\operatorname{Sh}\left(2^{X}\right) \geqslant \operatorname{Sh}\left(2^{X}\right)$ or $\operatorname{Sh}\left(2^{X}\right) \leqslant \operatorname{Sh}\left(2^{Y}\right)$ and also $\operatorname{Sh}(C(X)) \geqslant \operatorname{Sh}(C(Y))$ or $\operatorname{Sh}(C(X)) \leqslant \operatorname{Sh}(C(Y))$. Moreover, if both $\square X$ and $\square Y$ are infinite, then $\operatorname{Sh}\left(2^{X}\right) \equiv \operatorname{Sh}\left(2^{Y}\right)$, that is, $\operatorname{Sh}\left(2^{X}\right) \geqslant \operatorname{Sh}\left(2^{X}\right)$ and $\operatorname{Sh}\left(2^{X}\right) \leqslant \operatorname{Sh}\left(2^{X}\right)$

Proof. Since $X, Y \in \mathscr{M}, \square X$ and $\square Y$ are 0 -dimensional compact metric spaces. If $|\square X| \geqslant \kappa_{1}$, then $\square X$ contains a Cantor discontinuum. Hence $\square Y$ is embedded into $\square X$ so that $\square Y$ is a retract of $\square X$ (see for example [5, Theorem 4]) and $2^{\square Y}$ is a retract of $2^{\square X}$. Thus $\operatorname{Sh}(\square X) \geqslant \operatorname{Sh}(\square Y)$ and $\operatorname{Sh}\left(2^{\square X}\right) \geqslant \operatorname{Sh}\left(2^{\square X}\right)$. Therefore $\operatorname{Sh}(C(X)) \geqslant \operatorname{Sh}(C(Y))$ and $\operatorname{Sh}\left(2^{X}\right) \geqslant \operatorname{Sh}\left(2^{Y}\right)$ by Theorem 1. If $|\square X| \leqslant \kappa_{0}$ and $|\square Y| \leqslant s_{0}$, then $\square X$ and $\square Y$ are homeomorhic to ordered compacta by Mazurkiewicz and Sierpiński [12, Théorème, p. 21] and hence it holds that there is an embedding: $\square X \rightarrow \square Y$ or $\square Y \rightarrow \square X$. This completes the proof of the first part of the corollary. Next, let $\square X$ and $\square Y$ be infinite sets. By Pelczyński [15] both $2^{X}$ and $2^{X}$ contain Cantor discontinua. Hence there are embeddings: $2^{\square x} \rightarrow 2^{\square X}$ and $2^{\square Y} \rightarrow 2^{\square X}$ so that both the relations $\operatorname{Sh}\left(2^{\square X}\right) \geqslant \operatorname{Sh}\left(2^{\square Y}\right)$ and $\operatorname{Sh}\left(2^{\square X}\right) \leqslant \operatorname{Sh}\left(2^{\square Y}\right)$ hold. The corollary is a consequence of Theorem 1 .

Example 2. Let $X$ be a Cantor discontinuum and let $Y$ be a countably infinite compact set. Then $\operatorname{Sh}\left(2^{X}\right) \equiv \operatorname{Sh}\left(2^{Y}\right)$ by Corollary 5. However $\operatorname{Sh}\left(2^{X}\right) \neq \operatorname{Sh}\left(2^{Y}\right)$ because $2^{X}$ has no isolated points by Michael $[13,4.13 .4]$ and on the other hand $2^{Y}$ has isolated points (every isolated point of $Y$ is isolated in $2^{Y}$ ). This example shows that we can not replace the relation $\operatorname{Sh}\left(2^{X}\right) \equiv \operatorname{Sh}\left(2^{X}\right)$ in Corollary 5 by $\operatorname{Sh}\left(2^{X}\right)=\operatorname{Sh}\left(2^{Y}\right)$.

The following example shows that the hypothesis $X, Y \in \mathscr{M}$ in Corollary 5 is essential.

EXAMPLE 3. Let $X^{\prime}$ be a discrete space whose cardinality $\left|X^{\prime}\right|=\aleph_{1}{ }_{1}$ and let $X$ be a one point compactification of $X^{\prime}$. Next, let $D$ be a set consisting of exactly two points and let $Y=\prod_{\alpha \in \Omega} D_{\alpha}$, where $|\Omega|=\kappa_{1}$ and each $D_{\alpha}$ is a copy of $D$. Then both $X$ and $Y$ are 0 -dimensional compact spaces with an infinite number of points and hence $C(X)=X$ and $C(Y)=Y$. Since $Y$ has no isolated points, there is no embedding of $Y$ into $X$ so that $\operatorname{Sh}(X) \ngtr \operatorname{Sh}(Y)$. Suppose that $\operatorname{Sh}(X) \leqslant \operatorname{Sh}(Y)$. Then there is an embedding $i: X \rightarrow Y$ and a retraction $r: Y \rightarrow i(X)$ by Mardešić and Segal [8, Theorem 20]. Since $Y$ has Souslin property (= the countable chain condition) by Sanin [18], $i(X)$ must have Souslin property. This contradiction means $\operatorname{Sh}(X) \nless \operatorname{Sh}(Y)$. Finally, suppose $\operatorname{Sh}\left(2^{X}\right) \leqslant \operatorname{Sh}\left(2^{Y}\right)$. Then there is an embedding $i: 2^{X} \rightarrow 2^{Y}$ and a retraction $r: 2^{Y} \rightarrow i\left(2^{X}\right)$. Note that, by the definition of the finite topology, if $Z$ is separable then $2^{Z}$ is separable. Since $Y$ is separable by Ross and Stone [17], $2^{Y}$ is separable so that $2^{X}$ must be separable. However it is easy to see that each point of $X^{\prime}$ is isolated in $2^{X}$ and hence $2^{X}$ is not separable. This contradiction shows that $\operatorname{Sh}\left(2^{X}\right) \not \equiv \operatorname{Sh}\left(2^{Y}\right)$.

Theorem 3. Let $n$ be a positive integer. If $X$ and $Y$ are compact, then $\operatorname{Sh}(X) \leqslant \operatorname{Sh}(X) \quad($ resp. $\quad \operatorname{Sh}(X)=\operatorname{Sh}(Y))$ implies $\operatorname{Sh}(X(n)) \leqslant \operatorname{Sh}(Y(n)) \cdot($ resp. $\operatorname{Sh}(X(n))=\operatorname{Sh}(Y(n)))$.

Proof. Let $\underline{X}=\left\{X_{\alpha}, \pi_{\alpha}^{\beta}, \Omega\right\}$ and $\underline{Y}=\left\{Y_{\gamma}, \mu_{\gamma}^{\delta}, \Gamma\right\}$ be ANR-systems consisting of finite dimensional compact ANR's $X_{\alpha}$ and $Y_{\gamma}$ associated with $X$ and $Y$ respectively. Suppose that $\operatorname{Sh}(X) \leqslant \operatorname{Sh}(Y)$. There are maps $f: \underline{X} \rightarrow \underline{Y}$ and $\underline{g}: \underline{Y} \rightarrow \underline{X}$ such that $g f \simeq 1_{X}$. (See Mardešić and Segal [8] for notations.) Let $f=\left\{f_{\gamma}, \bar{\Gamma}\right\}$ and $\bar{g}=\left\{g_{\alpha}, \Omega\right\}$. For each $\alpha \in \Omega$ there is an index $\alpha^{\prime} \in \Omega$ such that $\alpha^{\prime}>f g(\alpha), \alpha$ and

$$
g_{\alpha} f_{g(\alpha)} \pi_{f g(\alpha)}^{\alpha^{\prime}} \simeq \pi_{\alpha}^{\alpha^{\prime}}: X_{\alpha^{\prime}} \rightarrow X_{\alpha} .
$$

Consider the systems $\underline{X}(n)=\left\{X_{\alpha}(n), \tilde{\pi}_{\alpha}^{\beta}, \Omega\right\}$ and $\underline{Y}(n)=\left\{Y_{\gamma}(n), \tilde{\mu}_{y}^{\delta}, \Gamma\right\}$, where $\tilde{\pi}_{\alpha}^{\beta}=\pi_{\alpha *}^{\beta} \mid X_{\beta}(n)$ and $\tilde{\mu}_{\gamma}^{\delta}=\mu_{\gamma *}^{\delta} \mid Y_{\delta}(n)$. By Lemmas 5 and $2, \underline{X}(n)$ and $\underline{Y}(n)$ are ANR-systems associated with $X(n)$ and $Y(n)$ respectively. For each $\gamma \in \bar{T}$, define 5 - Fundamenta Mathematicae C 
$f_{\gamma}(n): X_{f(\alpha)}(n) \rightarrow Y_{\gamma}(n)$ by $f_{\gamma}(n)=f_{\gamma *} \mid X_{f(\gamma)}(n)$ and similarly define $g_{\alpha}(n): Y_{g(\alpha)}(n)$ $\rightarrow X_{\alpha}(n), \alpha \in \Omega$, by $g_{\alpha}(n)=g_{\alpha *} \mid Y_{g(\alpha)}(n)$. By (3.6) and Lemma 1 it holds that

$$
g_{\alpha}(n) f_{g(\alpha)}(n) \tilde{\pi}_{f g(\alpha)}^{\alpha^{\prime}} \simeq \tilde{\pi}_{\alpha}^{\alpha^{\prime}}: X_{\alpha^{\prime}}(n) \rightarrow X_{\alpha}(n) .
$$

Also Lemma 1 shows that $f(n)=\left\{f_{\gamma}(n), \Gamma\right\}$ and $g(n)=\left\{g_{\alpha}(n), \Omega\right\}$ are maps of $\underline{X}(n)$ into $\underline{Y}(n)$ and of $\underline{Y}(n)$ into $\underline{X}(n)$ respectively. Since (3.7) implies $\underline{g}(n) f(n) \simeq 1_{\underline{x}}$, $\overline{\operatorname{Sh}}(X(n)) \leqslant \operatorname{Sh}(Y(n))$. That $\operatorname{Sh}(X)=\operatorname{Sh}(Y)$ implies $\operatorname{Sh}(X(n))=\operatorname{Sh}(Y(n))$ is proved similarly. hold.

COROLLARY 6. For a positive integer $n$ and a compact space $X$, the followings

(i) If $X$ is an ASR, then $X(n)$ is an ASR.

(ii) If $X$ is an ANSR, then $X(n)$ is an ANSR.

(iii) If $X$ is movable [7], then $X(n)$ is movable.

(iv) If $X$ is uniform movable [14], then $X(n)$ is uniform movable.

Proof. Suppose that $X$ is an ANSR. By Mardešic [10, Theorem 6] there is a finite dimensional compact ANR-space $Y$ such that $\operatorname{Sh}(X) \leqslant \operatorname{Sh}(Y)$. From Theorem 3 it follows that $\operatorname{Sh}(X(n)) \leqslant \operatorname{Sh}(Y(n))$. Since by Lemma $5 Y(n)$ is a compact ANR-space, by applying Mardešic [10, Theorem 6$]$ again we know $X(n)$ is an ANSR. The proof of (i) is similar. The assertions (iii) and (iv) are proved by the same argument as in the proof of Theorem 3.

\section{References}

[1] A. V. Arhangel'ski1, An additition theorem for weight of sets lying in bicompacta, Dokl. Akad. Nauk SSSR 126 (1959), pp. 239-241 (Russian).

[2] K. Borsuk, Theory of shape, Lecture Note Series 28, Math. Inst. Aarhus Univ. 1971.

[3] T. Ganea, Symmetrische Potenz topologisher Räume, Math. Nachr. 11 (1954), pp. 305-316.

[4] Y. Kodama, Mappings of a fully normal space into an absolute neighborhood retract, Sci. Rep. Tokyo Koiku Daigaku 5 (1955), pp. 37-47.

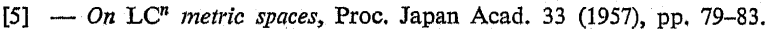

[6] - Decomposition spaces and shape of Fox, Fund. Math. 97 (1977), pp. 199-208.

[7] S. Mardešić and J. Segal, Movable compacta and ANR-systems, Bull. Acad. Polon. Sci. Sér. Sci. Math. Astronom. Phys. 18 (1970), pp. 649-654.

[8] - - Shapes of compacta and ANR-systems, Fund. Math. 72 (1971), pp. 41-59.

[9] - - Equivalence of Borsuk and the ANR-system approach to shapes, Fund. Math. 72 (1971), pp. 61-68.

[10] - Retracts in shape theory, Glasnik Math. 6 (1971), pp. 153-163.

[11] - Shapes for topological spaces, Gen. Topology and Appl. 3 (1973), pp. 265-282.

[12] S. Mazurkiewicz and W. Sierpiński, Contribution à la topologie des ensembles dénombrables, Fund. Math. 1 (1920), pp. 17-27.

[13] E. Michael, Topologies on spaces of subsets, Trans. Amer. Math. Soc. 71 (1951), pp. 152-181.

[14] M. Moszyńska, Uniformly movable compact spaces and their algebraic properties, Fund. Math. 77 (1972), pp. 125-144.

[15] A. Pelczyński, A remark on spaces $2^{X}$ for zerodimensional $X$, Bull. Acad. Polon. Sci. Sér. Sci. Math. Astronom. Phys. 13 (1965), pp. 85-89.
[16] V. I. Ponomarev, On continuous decompositions of bicompacta, Uspehi Math. Nauk (N. S.) 12 (1957), 4 (76), pp. 335-340; Amer. Math. Soc. Translation, Ser. 2, 30 (1963), pp. 235-240.

[17] K. A. Ross and A. H. Stone, Products of separable spaces, Amer. Math. Monthly 71 (1964), pp. $398-403$.

[18] N. H. Sanin, On intersection of open subsets in the product of topological spaces, C. R. (Doklady) Acad. Sci. URSS (N. S.) 53 (1946), pp. 499-501 (Russian).

[19] J. Segal, Hyperspaces of the inverse limit spaces, Proc. Amer. Math. Soc. 10 (1959), pp. 706-709.

[20] M. Wojdysławski, Rétractes absolus et hyperespaces des continus, Fund. Math. 32 (1939), pp. 184-192.

\section{DEPARTMENT OF MATHEMATICS}

UNIVERSTTY OF TSUKUBA

Ibaragi

DEPARTMENT OF MATHEMATICS

UNIVERSITY OF WARSAW

Warszawa

DEPARTMENT OF MATHEMATICS

TOKYO UNIVERSITY OF EDUCATION

Tokyo

Accepté par la Rédaction le 9. 2. 1976 